\title{
On the Effect of the Application of Traditional Chinese Medicine to Umbilicus with Timing Point Massage on Improving Gastrointestinal Function and Satisfaction Degree of Bedridden Patients in Department of Orthopedics
}

\author{
Wang Xiaohui, Xiao Bin, Zhang Xiaoxia, Teng Liang, Yin Chunlian \\ Wuwei Second People's Hospital of Gansu Province, Wuwe, Gansu Province, 733000
}

Keywords: application of traditional Chinese medicine to umbilicus; point massage; bedridden patient in Department of Orthopedics; gastrointestinal function; degree of satisfaction

\begin{abstract}
To explore the effect of the application of traditional Chinese medicine to umbilicus combined with timing point massage on improving gastrointestinal function and satisfaction degree of bedridden patients in Department of Orthopedics, 148 bedridden patients in orthopedics department of our hospital were divided into control group and observation group according to the method of turning over cards and drawing lots. 74 patients in control group were treated with routine therapy and 74 patients in observation group were treated with the application of traditional Chinese medicine to umbilicus combined with timing point massage. The clinical efficacy, improvement of gastrointestinal function and satisfaction with the treatment were compared between two groups. After treatment, the total effective rate of patients in observation group was significantly higher than that of control group $(\mathrm{P}<0.05)$. Besides, the time of first anus exhaust and defecation of observation group were earlier than that of control group and the former' incidence of abdominal distention were relatively lower $(\mathrm{P}<0.05)$. The treatment satisfaction of observation group was significantly higher than that of control group $(\mathrm{P}<0.05)$, and the incidence of adverse reactions of observation group was significantly lower than control group. These differences were statistically significant $(\mathrm{P}<0.05)$. The treatment of bedridden patients in department of orthopedics with application of traditional Chinese medicine to umbilicus combined with timing point massage can effectively improve their gastrointestinal function, reduce the complications of abdominal distension, improve the overall clinical efficacy and patients' satisfaction, as well as help them have an early recover.
\end{abstract}

As a result of prolonged ill time in bed combined with the trauma caused by anaesthesia and surgery, the local tissues of bedridden patients in department of orthopedics were severely oppressed and the blood circulation was obstructed, resulting in a severe malnutrition and serious effects on the stomach ${ }^{[1]}$. Because of slow gastrointestinal peristalsis, patients often have constipation, bringing a great pain ${ }^{[2]}$. Western medicine always applies cathartics in the treatment, such as Kaiselu, however this kind of treatment just can bring temporary medical relief, and the effect is not ideal ${ }^{[3]}$. In order to improve patients' gastrointestinal function as soon as possible, Theory of Traditional Chinese Medicine holds that applying traditional Chinese medicine of Evodia Rutaecarpa to umbilicus combined with point massage to patients will achieve to relieve their constipation and improve stomach function. To a certain extent, it can also reduce the incidence of abdominal distension ${ }^{[4]}$. In this study, 148 bedridden patients in department of orthopedics were divided into two groups, one of which receives the treatment of application of traditional Chinese medicine to umbilicus combined with timing point massage, and the results are shown in this paper.

\section{Clinical Data and Treatment Methods}

\subsection{Clinical Data}

All 148 patients including 83 males and 65 females in this study were from the orthopedic department of our hospital and were divided into two groups: the control group and the observation group. Each group had 74 patients, all of whom were aged 17-60. Their average age was 37.28 
\pm 6.13 . There was no difference in basic clinical data such as gender and age between these two groups ( $\mathrm{P}>0.05)$, and the specific conditions of two groups were shown in Table 1.

Table 1 Comparison of Basic Clinical Data between Two Groups (Case, Age)

\begin{tabular}{lllll}
\hline gender & $\begin{array}{l}\text { Control } \\
\text { Group }\end{array}$ & $\begin{array}{l}\text { Observation } \\
\text { Group }\end{array}$ & $t / x^{2}$ & $P$ \\
\hline Male & $43(58.11)$ & $42(56.76)$ & 0.028 & 0.868 \\
Female & $31(41.89)$ & $32(43.24)$ & 0.028 & 0.868 \\
Average Age & $36.79 \pm 7.32$ & $37.10 \pm 5.23$ & 0.296 & 0.767 \\
\hline
\end{tabular}

\subsection{Exclusion and Inclusion Criteria}

Patients' inclusion criteria: (1) All patients had rest in bed for 14 days after orthopedics operation; (2) All patients agreed to participate in the study, and their families supported for signing the consent form; (3) All patients had gastrointestinal discomfort and constipation, etc.; (4) All patients had normal communication and no language loss. Exclusion criteria: 1) Patients suffered from severe heart disease; 2) Patients with severe mental illness or neuroschism; 3) Patients with severe vascular injury in lower extremities; 4) Patients with poor compliance.

\subsection{Treatment}

Patients in control group received routine treatment after orthopedics operation and intramuscular injection of 100mg vitamin B1 (Manufacturer: Jiangsu Fang Qiang Pharmaceutical Factory, Specification: 2ml:50mg, Permission Number: 20020429), so as to promote intestinal peristalsis and improve gastrointestinal function. Utility time: once per day, lasting 3 days. During the recovery period after operation, patients were guided to take the exercise of limbs and prevent constipation.

The observation group patients were treated with the application of traditional Chinese medicine to umbilicus combined with timing point massage after operation. (1) Application of traditional Chinese medicine to umbilicus: during the process of application of traditional Chinese medicine to umbilicus, we need to pay attention keep patients warm and the skin condition around umbilicus at all times, adjust patients' diet, and prevent stomach flatulence. The prescription of applying traditional Chinese medicine to umbilicus is as follows: 12hours after patients' operation, we took 3-5g Evodia Rutaecarpa, ground it to fine powder, added $5 \mathrm{ml}$ vinegar and adjusted it into paste, and then heated it to $40{ }^{\circ} \mathrm{C}$. It was necessary to clean patients' umbilicus with physiological saline, then spread the heated Evodia Rutaecarpa out on two layers of square gauze, the thickness of which was about $0.5 \mathrm{~cm}$. Then the gauze was folded around and applied to the patients' umbilical fossa. The gauze was fixed with a rubber cloth and changed every 12 hours, which should last 3 days. (2) Abdominal point massage: professional personnel introduced the detailed massages of massage method and the position of acupoints to the patients and their families. Only with the obtainment of the consent of patients can they carry out the point massage. During the massage, professional personnel paid attention to keep patients warm and prevented them from catching cold. The specific operation of point massage is as follows: patients took supine position, maintained even breathing, and relaxed abdominal muscles. Finger massage was taken which centered four main points which include Bilateral Zusanli Point which is 3 inches below the knees and $1.5 \mathrm{~cm}$ beside the lateral tibia, Tianshu Point which is 2 inches by the side of the umbilicus, Guilai Point which is 4 inches below the middle of umbilicus and 2 inches beside the anterior median line, and Zhigou Point between the ulnar and radius which is 3 inches on the wrist in the back of the hand. Patients bent his two knees, located Sanyinjiao Point and Zusanli Point between right thumbs, and applied his hands vertically downward on the points he had found. After two minutes of pressing the Tianshu Point with the middle fingers, we gradually and clockwisely increased the strength from the right lower abdomen. When patients' abdomen produced the acid-swelling feeling, then the massage effects achieved the 
best condition. Point massage was better in 1 hour after meal, and massage time of each point was about 3-5 minutes which was twice in every morning and evening, until patients' anal gas discharged.

\subsection{Observation Index}

(1) Observe the clinical efficacy of two groups. (2) Observe the improvement of the stomach and intestines of two groups, including recording the time of first anal exhaust and first anal defecation of two groups. (3) Record the occurrence of abdominal distention in two groups, among which, the incidence of abdominal distension was statistically analyzed according to patients' main complainta. (4) Observe the treatment satisfaction of two groups and evaluate the treatment through satisfaction questionnaire. The total score was 100 points and higher the score, higher the satisfaction. (5) Record the adverse reactions of two groups, including bedsore, muscle atrophy, joint stiffness, skin hypersensitivity and other complications.

\subsection{Evaluation Standard of Curative Effect}

This study evaluated the clinical efficacy of two groups according to The Criteria for the Diagnosis of TCM Symptoms issued by the Chinese Medicine Administration in $1994{ }^{[5]}$. Within 1 day after medical application, anus exhaust would appear, defecation was smooth, bowel sounds were loud, active time was more than 10 minutes, and there was no abdominal distention, so the treatment was effective. Within $1 \sim 3$ days, patients had anal exhaust, unsmooth defecation, recovered bowel sounds and effective abatement of abdominal distention, so the treatment was effective. After 3 days, there was no anal exhaust and no change in clinical symptoms, so the treatment was effective. Total clinical efficiency= (number of notable cases + number of valid cases) / total number of cases $\times 100 \%$.

\subsection{Statistical Method}

All the data of this study was input into SPSS18.0 software, and the statistical analysis has been carried out, in which the measurement data was all described with $(\bar{X} \pm \mathrm{s})$ and the difference between groups was tested with t. Moreover, the counting data was described by "ratio (\%)", and tested with $\mathrm{x} 2$, when $\mathrm{P}<0.05$, the difference is statistically significant.

\section{Results}

\subsection{Comparison of Clinical Efficacy between Two Groups}

After treatment, the total effective rate of patients in observation group was significantly higher than that of control group. The difference between two groups was statistically significant $(\mathrm{P}<$ 0.05 ), as shown in Table 2.

Table 2 Comparison of Clinical Efficacy between Two Groups

\begin{tabular}{|c|c|c|c|c|c|}
\hline Group & $\begin{array}{l}\text { Number of } \\
\text { Cases }\end{array}$ & notable & effective & ineffective & $\begin{array}{l}\text { total } \\
\text { effective } \\
\text { rate }\end{array}$ \\
\hline $\begin{array}{l}\text { Control } \\
\text { Group }\end{array}$ & 74 & $11(14.86)$ & $45(60.81)$ & $18(24.32)$ & $56(75.68)$ \\
\hline $\begin{array}{l}\text { Observation } \\
\text { Group }\end{array}$ & 74 & $39(52.70)$ & $34(45.95)$ & $1(1.35)$ & 73(98.65) \\
\hline$t$ & - & - & & & 15.458 \\
\hline$P$ & - & - & & & 0.000 \\
\hline
\end{tabular}




\subsection{Comparison of the Improvement of Gastrointestinal Function between Two Groups}

After treatment, the time of first anal exhaust and the first anal defecation in observation group was significantly shorter than those in control group, and the difference was significant $(\mathrm{P}<0.05)$, as shown in Table 3.

Table 3 Comparison of the Improvement of Gastrointestinal Function between Two Groups [( $\bar{X} \pm \mathrm{s})$, h]

\begin{tabular}{llll}
\hline Group & $\begin{array}{l}\text { Number of } \\
\text { Cases }\end{array}$ & First Anal Exhaust Time & First Anal Defecation Time \\
\hline $\begin{array}{l}\text { Control Group } \\
\text { Observation }\end{array}$ & 74 & $53.29 \pm 23.21$ & $67.84 \pm 24.12$ \\
Group & 74 & $23.31 \pm 12.19$ & $37.92 \pm 17.33$ \\
$X^{2}$ & - & 9.873 & 8.666 \\
$P$ & - & 0.000 & 0.000 \\
\hline
\end{tabular}

\subsection{Comparison of the Incidence Rate of Abdominal Distension between Two Groups}

After treatment, 10 patients in control group had mild abdominal distension and 3 patients had severe abdominal distension, so the incidence of abdominal distension was $17.57 \%$ (13/74). In observation group, only two patients had mild abdominal distension, and the other patients had normal curative effects, so the incidence of abdominal distension was $2.70 \%$. After comparison, the incidence of abdominal distention in observation group was significantly lower than that in control group ( $\mathrm{X} 2=7.416, \mathrm{P}=0.006)$.

\subsection{Comparison of Patients' Satisfaction Degree between Two Groups}

After treatment, patients' satisfaction degree in observation group was significantly higher than that in control group. The difference between two groups was statistically significant $(\mathrm{P}<0.05)$, as shown in Table 4.

Table 4 Comparison of Patients’ Satisfaction Degree between Two Groups Cases (\%)

\begin{tabular}{|c|c|c|c|c|c|}
\hline Group & $\begin{array}{l}\text { Number of } \\
\text { Cases }\end{array}$ & Satisfied & General & Unsatisfied & $\begin{array}{l}\text { Total } \\
\text { Satisfaction }\end{array}$ \\
\hline $\begin{array}{l}\text { Control } \\
\text { Group }\end{array}$ & 74 & $19(25.68)$ & $36(48.65)$ & $19(25.68)$ & $55(74.32)$ \\
\hline $\begin{array}{l}\text { Observation } \\
\text { Group }\end{array}$ & 74 & $38(51.35)$ & $33(44.59)$ & $3(4.05)$ & 71(95.95) \\
\hline$t$ & - & - & & & 12.013 \\
\hline$P$ & - & - & & & 0.001 \\
\hline
\end{tabular}

\subsection{Comparison of the Incidence Rate of Adverse Reactions between Two Groups}

After treatment, there were 11 cases of bedsore, 3 cases of joint stiffness and 1 case of muscular atrophy in control group, so the adverse reaction rate was $20.27 \%(15 / 74)$. While in observation group, there was only 1 case of skin allergy around the umbilicus, and none of the other patients had abnormal symptoms, so the adverse reaction rate was $1.35 \%$ (1/74). After comparison, incidence rate of adverse reactions in observation group was significantly lower than that in control group $(\mathrm{X} 2=11.843, \mathrm{P}=0.001)$. 


\section{Conclusion}

For patients in orthopedic department, within 1 day after orthopedics surgery, their gastrointestinal peristalsis often disappears indirectly, and gradually the gas is expelled from the body over time. If patients are unable to remove the gas from the body by himself after surgery, it will cause abdominal expansion. As a result, the diaphragm movement of patients is severely restricted, which destroys the exhaust operation of body, leads to the obstruction of gastrointestinal function and affects the wound healing ${ }^{[6]}$. According to the Theory of Traditional Chinese Medicine, this study takes the application of traditional Chinese medicine to umbilicus combined with timing point massage to treat bedridden patients after orthopedics operation. The clinical efficacy is very remarkable ${ }^{[7]}$.

Umbilicus is also called Shenque Point. In embryonic development, umbilicus is the last closed place, belonging to the most important points of Ren Meridian. Umbilicus contains a large number of nerves, Ren Meridian, Chong Vessel and Governor Vessel ${ }^{[8]}$. Evodia Rutaecarpa has the effect of liver soothing and ventilation as well as pain-relieving. Umbilicus connects 12 meridians, connecting the internal organs, so it is the total pivot of General armature of meridians and collaterals, which can reach the purpose of regulating and treating hundreds of diseases ${ }^{[9]}$. By applying Evodia Rutaecarpa to umbilicus to treat bedridden patients, it can dredge the channels and collaterals, so as to promote the gastrointestinal peristalsis and moisturize the intestines and defecation ${ }^{[10]}$. Evodia Rutaecarpa has the effect of relieving pain, while vinegar can accelerate the effective absorption of drug components. The interaction between them can promote the enhancement of gastrointestinal motility and improve the gastrointestinal function of bedridden patients ${ }^{[11]}$.

By massage four main points of Zusanli, Tianshu, Guilai and Zhigou, we can clear the viscera and eliminate constipation ${ }^{[12]}$. Theory of Traditional Chinese Medicine believes that Zusanli belongs to the Yangming Stomach Meridian Point, which mainly treats gastrointestinal syndrome. Tianshu is the Hand Yangming Large Intestine Meridian Point, which mainly treats stomach pain and abdominal distension. Guilai locates in the lower abdomen, mainly treating abdominal pain and hernia. Zhigou is one of the common points of Shaoyang Sanjiao Meridian of Hand that focus on the treatment of constipation ${ }^{[13]}$. Meanwhile, by massaging the abdominal points, we can invigorate the spleen and stomach, thus stimulate hormone enterogastric peristalsis, improve the viscera function, promote the intestinal blood circulation, and increase the Intestinal mucosal neuronutrition ${ }^{[14]}$. Through clockwise massage on the abdomen, it can directly stimulate the gastrointestinal tract, increase the peristaltic rhythm among the small and big intestines and make stool softened and easy to expel ${ }^{[15]}$.

The results of this study show that the total efficacy rate of patients in observation group after treatment is significantly higher than that in control group. It is suggested that the application of traditional Chinese medicine to umbilicus combined with timing point massage can effectively improve the clinical efficacy, alleviate the pain of patients, and improve their satisfaction degree. Compared with control group, the first anal exhaust and defecation time in observation group were earlier and the incidence of abdominal distension and adverse reactions were also relatively lower. Therefore, the application of traditional Chinese medicine to umbilicus can effectively improve the function of intestines and stomach of patients. The combination of it with point massage has multiple effects. By stimulating the peristalsis of large intestine, we can accelerate a rapid exhalation of anus after operation, make defecate smoothly and shorten the first defecation time. This will also prevent effectively abdominal distension and other complications, reduce the incidence of adverse reactions to a certain extent, and help patients recover quickly.

To sum up, it can effectively improve the gastrointestinal function of patients in orthopedics department after orthopedic surgery by applying traditional Chinese medicine to umbilicus combined with timing point massage, which is also simple and safe. Patients will have less complications, high degree of satisfaction, and good clinical effects. It also can effectively prevent the occurrence of postoperative abdominal distension which is conducive to the rehabilitation of patients, therefore, clinical application is worth popularizing. 


\section{References}

[1] Yu Jiaoyang. Effect of Application of Evodia Rutaecarpa to Umbilicus Combined with Timing Point Massage on the Recovery of Gastrointestinal Function of Elderly Patients after Orthopedics Operation [J]. Pharmaceutical Journal of Chinese People's Liberation Army, 2016,32(01):74-76.

[2] Wang Xiaohui. Clinical Efficacy Observation on Improving Gastrointestinal Function of Bedridden Patients in Department of Orthopedics with the Application of Traditional Chinese Medicine to Umbilicus Combined with Timing Point Massage [J]. China Health Care \& Nutrition, 2017,27(4):30.

[3] Sun Xinying. Prevention and Clinical Efficacy Observation of Integrated Nursing Care of Traditional Chinese and Western Medicine on Gastrointestinal Dysfunction after Abdominal Operation [J]. Electronic Journal of Cardiovascular Diseases with Integrated Chinese and Western Medicine, 2017,5(05):58-59.

[4] Ding Yuxi, Li Shurong. Effect of Point and Abdominal Massage on Urinary Retention and Gastrointestinal Dysfunction after Gynecologic Laparoscopic Surgery [J]. Journal of Liaoning University of Traditional Chinese Medicine, 2016,18(08):245-247.

[5] Zhou Yuan. Clinical Implementation and Effect Evaluation of Nursing Program of Traditional Chinese Medicine for Patients with Epigastric Pain Due to Eeficiency of Spleen and Stomach [D]. Guangxi University of Traditional Chinese Medicine, 2016.

[6] Xin Zhuang, Jie Ouyang, Chuntao Jiang, Qingsheng Liu. New. Approach to Develop A 3D Non-isothermal Computational Framework for Injection Molding Process Based on Level Set Method [J]. Chinese Journal of Chemical Engineering, 2016,24(07):832-842.

[7] Zhang Junhong, Jung Ruifang. Nursing of Constipation in Bedridden Patients in Department of Orthopedics [J]. Hebei Medicine, 2014 24: 2879-2880.

[8] Hossein E. Jazayeri, Martin Rodriguez-Romero, Mehdi Razavi, Mohammadreza Tahriri, Karan Ganjawalla, Morteza Rasoulianboroujeni, Mohammad H. Malekoshoaraie, Kimia Khoshroo, Lobat Tayebi. The Cross-Disciplinary Emergence of 3D Printed Bioceramic Scaffolds in Orthopedic Bioengineering[J]. Ceramics International, 2017,09(10):95-106.

[9] Huang Lili, Su Lin, Wu Haixia, et al. Therapeutic Effect of Warm Water Foot Bath Combined with Point Massage on the Recovery of Gastrointestinal Function of Patients after Postoperative Gastric Cancer Operation [J]. Medical Journal of National Defending Forces in Northwest China, 2015,36(09):612-613.

[10] Madalina Saracutu, Jaynie Rance, Helen Davies, Darren J. Edwards. Effects of Osteopathic Treatment on Psychosocial Factors in People with Persistent Pain: A Systematic Review[J]. International Journal of Osteopathic Medicine, 2018,27.

[11] Chan Sau-Man Conny, Ip Wan-Yim. The Effectiveness of Nurse-Led Preoperative Assessment Clinics for Patients Receiving Elective Orthopaedic Surgery: A Systematic Review[J]. Journal of Peri Anesthesia Nursing, 2016,31(06):465-474.

[12] Xiong Ya-qin. Effect of Point Massage Plus Fragrant Abdominal Ointment on Gastrointestinal Function after Esophageal Cancer Operation [J]. Shenzhen Journal of Integrated Traditional Chinese and Western Medicine, 2015,25(15):53-54.

[13] Liu Fen. Fennel Hot Compress Plus Point Massage Promote Anal Exhaust after Obstetrics and Gynecology Surgery [J]. Journal of Clinical Medical Literature, 2014,1 (13): 2407-2408.

[14] Zhang Le. Effect of Orthopedics Nursing in Psychological Nursing Combined with Humanized Nursing [J]. Chinese Journal of Management in Chinese Medicine, 2018,26 (07): 70-71.

[15] Ge Qinmei, Chen Junbo, Wu Jiayan. Effect of Implementation of Safety Link Management in Patients in Department of Orthopedics [J]. Chinese Journal of Management in Chinese Medicine, 2018,26 (07): 143-145. 